\title{
Limits on contact interactions and leptoquarks at HERA
}

\author{
Aleksander Filip Żarnecki* on behalf of the ZEUS Collaboration \\ Faculty of Physics, University of Warsaw \\ E-mail: Filip.Zarnecki@fuw.edu.pl
}

\begin{abstract}
High-precision HERA data corresponding to a luminosity of around $1 \mathrm{fb}^{-1}$ have been used in the framework of eeqq contact interactions (CI) to set limits on possible high-energy contributions beyond the Standard Model to electron-quark scattering. Measurements of the inclusive deep inelastic cross sections in neutral and charged current ep scattering were considered. The analysis of the $e p$ data has been based on simultaneous fits of parton distribution functions including contributions of CI couplings to ep scattering. Several general CI models and scenarios with heavy leptoquarks were considered. Improvements in the description of the inclusive HERA data were obtained for a few models. Since a statistically significant deviation from the Standard Model cannot be established, limits in the TeV range were set on all models considered.
\end{abstract}

European Physical Society Conference on High Energy Physics - EPS-HEP2019 -

10-17 July, 2019

Ghent, Belgium

${ }^{*}$ Speaker. 


\section{Introduction}

Precise knowledge of the parton densities inside the proton is crucial, in particular, for the full exploitation of the physics potential of the LHC. The H1 and ZEUS collaborations combined all inclusive data on $e^{ \pm} p$ scattering at HERA [1] and the resulting inclusive cross sections are widely used to extract parton distribution functions (PDFs) of the proton within the DGLAP formalism. The PDF set resulting from the HERA analysis [1] is denoted as HERAPDF2.0.

However, HERA measurements of deep inelastic $e^{ \pm} p$ scattering (DIS) cross sections at the highest values of negative four-momentum-transfer squared, $Q^{2}$, are also sensitive to different beyond the Standard Model (BSM) contributions even at scales far beyond the centre-of-mass energy of $320 \mathrm{GeV}$. If BSM physics effects existed in the HERA data, the current PDF sets would have been biased by absorbing unrecognized BSM contributions. Also, PDF uncertainties estimated within the SM analysis would have been significantly underestimated. A new approach was therefore developed, based on simultaneous fits of the PDFs and the contributions of "new physics" processes. The new procedure was first introduced by the ZEUS collaboration to set limits on the quark radius [2] and then extended to other "new physics" scenarios [3], which can be described within the contact-interaction (CI) framework. Summarised in this contribution are results presented in [3].

\section{Models for new physics}

Four-fermion CIs represent an effective theory which describes low-energy effects due to physics at much higher energy scales. Contact-interaction models can describe the effects of heavy leptoquarks, additional heavy weak bosons and electron or quark compositeness. The CI approach is not renormalisable and is only valid in the low-energy limit, far below the mass scale of the new physics. Vector CI currents considered here are represented by additional terms in the SM Lagrangian:

$$
\mathscr{L}_{\mathrm{CI}}=\sum_{\substack{i, j=L, R \\ q=u, d, s, c, b, t}} \eta_{i j}^{e q}\left(\bar{e}_{i} \gamma^{\mu} e_{i}\right)\left(\bar{q}_{j} \gamma_{\mu} q_{j}\right),
$$

where the sum runs over electron and quark chiralities and quark flavours. The couplings $\eta_{i j}^{e q}$ describe the chiral and flavour structure of CIs. It was assumed that the same coupling structure applies to all quarks. The one-parameter scenarios considered in the presented study are defined by sets of four coefficients, $\varepsilon_{i j}$, each of which may take the values \pm 1 or zero, and the coupling strength, $\eta$, or compositeness scale, $\Lambda$ :

$$
\eta_{i j}^{e q}=\eta \varepsilon_{i j}= \pm \frac{4 \pi}{\Lambda^{2}} \varepsilon_{i j}
$$

Note that the coupling strength $\eta$ can be both positive and negative, and the two cases are distinct because of the interference with the SM amplitudes. When setting limits for BSM contributions, scenarios with positive and negative $\eta$ values were considered separately.

Vector-type eeqq CI can be also used to describe effects of $s$ - and $t$-channel exchange of heavy leptoquarks (LQs) ${ }^{1}$. According to the general classification proposed by Buchmüller, Rückl and

\footnotetext{
${ }^{1}$ For the invariant mass range accessible at HERA, with $\sqrt{s} \approx 320 \mathrm{GeV}$, the heavy LQ approximation is already applicable for $M_{\mathrm{LQ}}>400 \mathrm{GeV}[6]$.
} 
Wyler [4], there are 14 possible LQ types (isospin singlets or multiplets): seven scalar and seven vector $^{2}$. The effective LQ coupling, $\eta_{\mathrm{LQ}}$, is given by the square of the ratio of the leptoquark Yukawa coupling, $\lambda_{\mathrm{LQ}}$, to the leptoquark mass, $M_{\mathrm{LQ}}$. The CI couplings of the Lagrangian (Eq. 2.1), $\eta_{i j}^{e q}$, can be then written as

$$
\eta_{i j}^{e q}=a_{i j}^{e q} \cdot \eta_{\mathrm{LQ}}=a_{i j}^{e q}\left(\frac{\lambda_{\mathrm{LQ}}}{M_{\mathrm{LQ}}}\right)^{2}
$$

where the coefficients $a_{i j}^{e q}$ depend on the LQ species and are twice as large for vector as for scalar leptoquarks. By definition, the values of $\eta_{\mathrm{LQ}}$ are positive.

\section{Extended fit to the inclusive HERA data}

The analysis follows the approach adopted for the determination of HERAPDF2.0 [1], based on a comparison of the measured inclusive cross sections with the model predictions, but is extended to take into account the possible BSM contributions, as described in [2,3]. The effects of each CI scenario are taken into account by scaling the NLO QCD predictions at given values of $x$ and $Q^{2}$, corresponding to the inclusive cross-section measurements [1], with the ratio of SM+CI and SM cross sections calculated in leading order in electroweak and CI couplings. The PDFs of the proton are described at a starting scale of $1.9 \mathrm{GeV}^{2}$ in terms of 14 parameters. These parameters, together with the considered systematic shifts of the cross sections and the possible contribution of BSM phenomena (described by the CI coupling $\eta$ ) were fitted to the data using a $\chi^{2}$ method. All fits were performed within the xFitter framework [7] modified to include CI contributions. Systematic uncertainties of the fit are also esimated following the approach described in [1]. Considered are the uncertainties due to the choice of the form of the parameterisation and due to the variation of model parameter settings.

When not taking into account the CI contribution, the resulting sets of PDFs, referred to as ZCIPDFs in the following, are in good agreement with HERAPDF2.0 fit results. For six out of 13 considered CI scenarios and seven out of 14 heavy-LQ models, no significant improvement in description of the data was observed when the ZCIPDF fit is extended by adding the CI coupling, $\eta$ (or $\eta_{\mathrm{LQ}}$ for LQ models) as an additional fit parameter. However, there are also four models (three $\mathrm{CI}$ and one LQ scenario), which result in an improved description of the data, with $\Delta \chi^{2}<-4$. The best description of the inclusive HERA data is obtained for the X6 model $\left(\Delta \chi^{2}=-6.01\right)$ and $S_{1}^{L}$ model $\left(\Delta \chi^{2}=-11.10\right)$. The fit results for these models are compared with HERA NC DIS data in Fig.1. Also indicated is the SM contribution to the NC DIS cross sections obtained from the QCD+CI fit. Figure 1 (left) shows that, for the X6 model, the determination of the proton PDFs is affected very little by the CI contribution; the SM part of the NC DIS cross sections extracted from the QCD+CI fit agree with the nominal ZCIPDF fit within the quoted PDF uncertainties. The situation is different for the $S_{1}^{L}$ heavy-LQ model shown in Fig.1 (right), where the description of the proton PDFs is significantly affected when the heavy-LQ contribution is taken into account in the fit. As a result, the cross-section prediction for NC $e^{+} p$ DIS due to $\gamma / Z^{0}$ exchange increases at the highest values of $Q^{2}, Q^{2}>50000 \mathrm{GeV}^{2}$, by about a factor of two.

\footnotetext{
${ }^{2}$ Leptoquark states are named according to the so-called Aachen notation [5].
} 

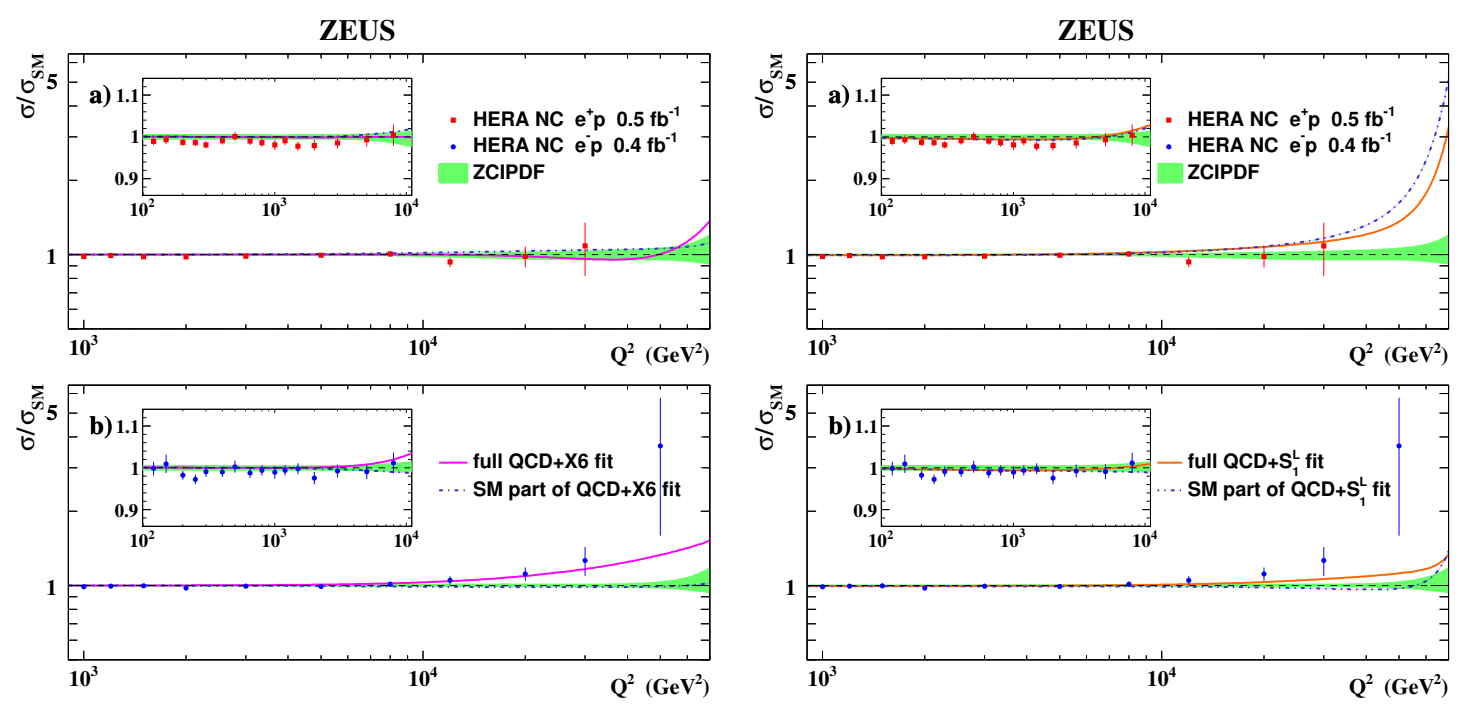

Figure 1: Result of the simultaneous QCD+CI fit to the HERA inclusive data, for the X6 CI model (left) and for the $S_{1}^{L}$ LQ model in the contact interaction limit, $M_{L Q} \gg \sqrt{s}$ (right), compared to the combined HERA (a) $e^{+} p$ and (b) $e^{-} p$ NC DIS data, relative to the SM expectations based on the QCD fit without the CI contribution (ZCIPDF). The bands represent the total uncertainty of the SM expectations. Also shown is the SM contribution to the cross section resulting from the combined fit. Figures taken from [3].

\section{Limit-setting precedure}

The limits on the mass scales of the $\mathrm{CI}$ and heavy-LQ models were derived in a frequentist approach [9] using the technique of replicas. Replicas are sets of cross-section values, corresponding to the HERA inclusive data set that are generated by varying all cross sections randomly according to their known uncertainties. To facilitate efficient processing of replica data, a simplified QCD fit method, based on the Taylor expansion of the cross-section predictions in terms of PDF parameters was developed [8]. The fitted $\eta$ values from the large sets of replicas, $\eta^{\text {Fit }}$, were used as a test statistics and compared to the corresponding value $\eta^{\text {Data }}$ determined from a fit to the data. The upper (lower) 95\% C.L. limit on the positive (negative) coupling, for a given scenario was determined as the true value of $\eta$ for which $95 \%$ of the replicas produced a fitted coupling value, $\eta^{\mathrm{Fit}}$, larger (smaller) than that found in the data, $\eta^{\text {Data }}$. To quantify the statistical consistency of the fit results with the SM expectations, the probability $p_{\mathrm{SM}}$ that an experiment assuming the validity of the SM would produce a value of $\eta^{\text {Fit }}$ greater than (or less than, for scenarios with negative $\eta$ ) that obtained from the data is also calculated.

\section{Results}

For six CI models (LR, RL, VV, X2, X4 and X5), $p_{\mathrm{SM}}$ is above $20 \%$, corresponding to less than a $1 \sigma$ deviation from the nominal fit result $\left(\eta^{\mathrm{Fit}}=\eta^{\mathrm{SM}} \equiv 0\right)$. For four models (LL, LR, VA and $\mathrm{X} 3$ ), the data fit results are reproduced by the SM replicas with 3-7\% probability, corresponding to about a $2 \sigma$ difference. However, for the three scenarios (AA, X1 and X6) with $\Delta \chi^{2}<-4, p_{\mathrm{SM}}$ is below $1 \%$. This confirms that the differences between the HERA data and the SM predictions 


\section{ZEUS}

HERA $\mathrm{e}^{ \pm} \mathrm{p}$ 1994-2007 95\% C.L. Limits

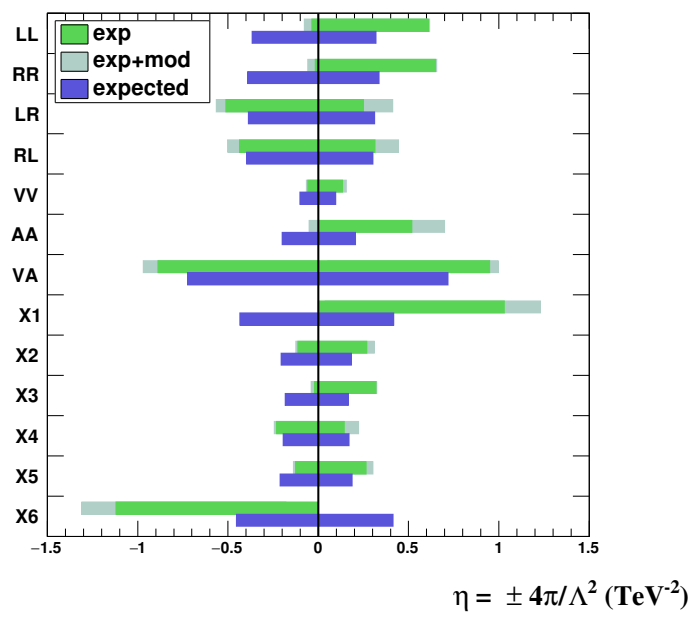

ZEUS

HERA $\mathrm{e}^{ \pm} \mathbf{p}$ 1994-2007 95\% C.L. Limits

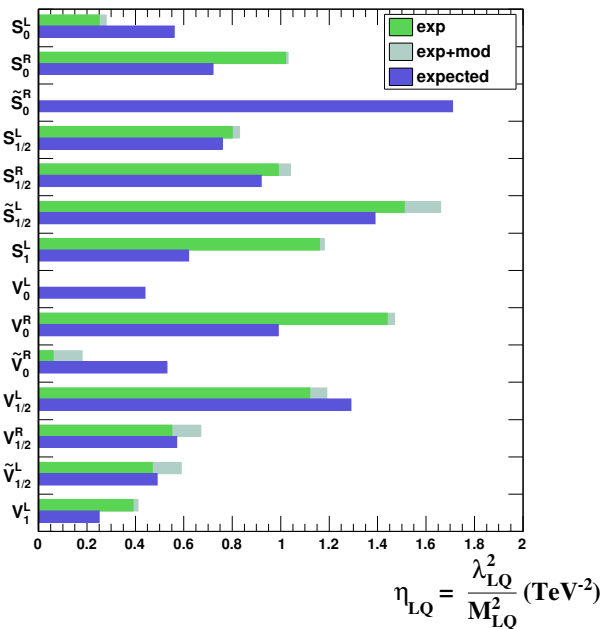

Figure 2: Limits on the CI coupling strength, $\eta= \pm 4 \pi / \Lambda^{2}$, evaluated at 95\% C.L. (left) and upper 95\% C.L. limits on the LQ coupling strength, $\eta_{\mathrm{LQ}}=\lambda_{\mathrm{LQ}}^{2} / M_{\mathrm{LQ}}^{2}$. Compared are the limits calculated without (dark upper bars) and with (light upper bars) modelling uncertainties, and the expected limits (lower bars). Figures taken from [3].

described by the additional CI contribution in the fit are unlikely to be due to statistical fluctuations only.

CI coupling limits calculated without (exp) and with (exp+mod) model and parameterisation variations, are compared to the expected limits in Fig.2 (left). Corresponding CI mass-scale limits, calculated taking modelling uncertainties into account, range from $3.1 \mathrm{TeV}$ for the $\mathrm{X} 6$ model $\left(\Lambda^{-}\right)$ up to $17.9 \mathrm{TeV}$ for the $\mathrm{X} 3$ model $\left(\Lambda^{-}\right)$. For the three models mentioned above (AA, X1 and X6), when only experimental uncertainties are considered, one sign of the CI coupling is excluded at 95\% C.L. and the limits for the coupling and compositeness scale $\Lambda$ are presented only for the other sign. The effect also persists when modelling uncertainties are taken into account for the X1 and X6 scenarios.

As already mentioned above, an improvement in the description of the HERA data can be obtained for the $S_{1}^{L}$ model, and the probability of reproducing the fit result with SM replicas, $p_{\mathrm{SM}}$, is below $0.01 \%$. For the $V_{0}^{R}$ model, the probability $p_{\mathrm{SM}}$ is $1.8 \%$, which means that for both models $\eta_{\mathrm{LQ}}=0$ is excluded at $95 \%$ C.L. When modelling uncertainties are taken into account, the corresponding $p_{\mathrm{SM}}$ values increase, but are still below 5\% for both models. For these two models the coupling limits extracted from the analysis of HERA inclusive data are much weaker than the expected limits. Coupling limits for different LQ scenarios, calculated without (exp) and with (exp+mod) model and parameterisation variations, are compared with the expected 95\% C.L. limits on $\lambda_{\mathrm{LQ}} / M_{\mathrm{LQ}}$ in Fig.2 (right).

In Fig.3, the measured $Q^{2}$ spectra of the HERA $e^{+} p$ and $e^{-} p$ data, relative to the SM predictions calculated using ZCIPDF, are compared with the expectations for the VV and AA contactinteraction models and for the $S_{1}^{L}$ and $V_{0}^{R}$ leptoquark models (as examples) which correspond to the limits shown in Fig.2. 
ZEUS
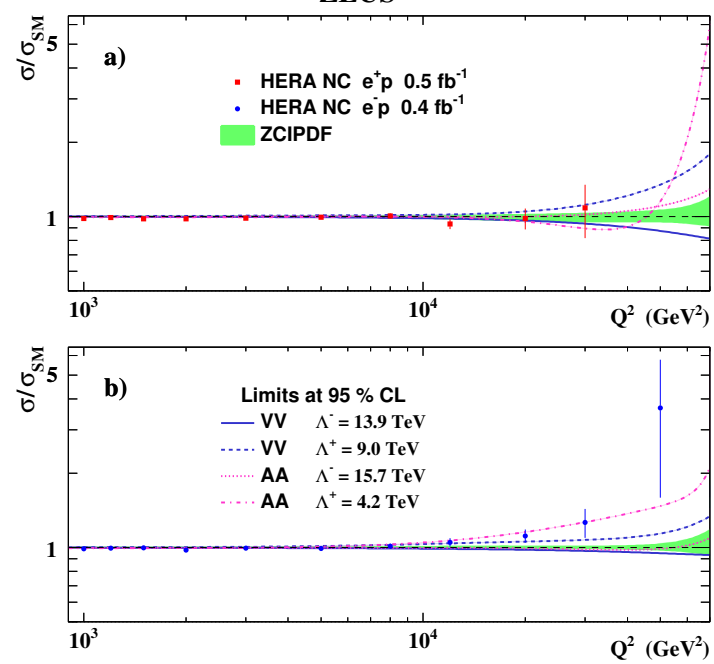

ZEUS
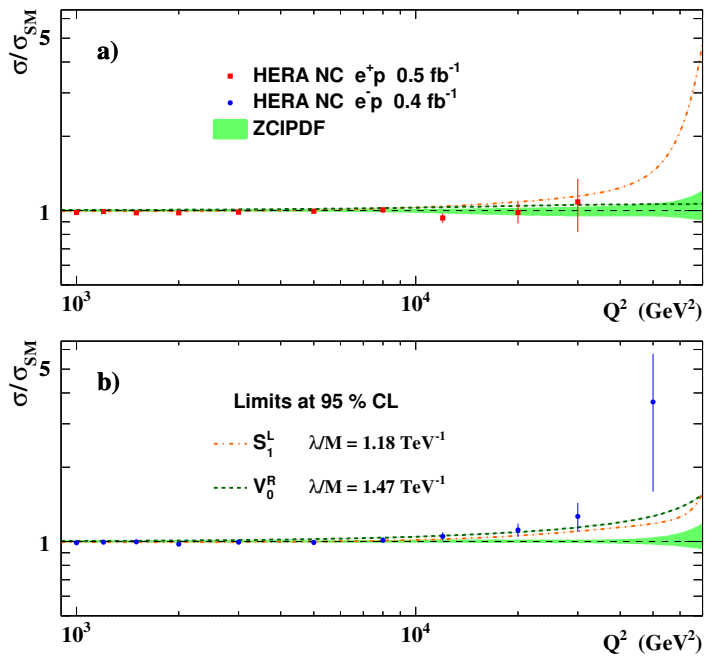

Figure 3: HERA (a) $e^{+} p$ and (b) $e^{-} p$ NC DIS data, relative to the SM expectations based on the ZCIPDF fit to the HERA inclusive data, compared to expectations from the VV and AA contact-interaction models (left) and from the $S_{1}^{L}$ and $V_{0}^{R}$ leptoquark models (right) with the effective couplings corresponding to the respective 95\% C.L. limits. The same models are shown on upper and lower plots. The bands represent the total uncertainty on the ZCIPDF fit predictions. Figures taken from [3].

\section{References}

[1] H1 and ZEUS Collaborations, H. Abramowicz et al., Combination of Measurements of Inclusive Deep Inelastic $e^{ \pm} p$ Scattering Cross Sections and QCD Analysis of HERA data, Eur. Phys. J. C 75 (2015) 580 [arXiv: 1506.06042$]$.

[2] ZEUS Collaboration, H. Abramowicz et al., Limits on the effective quark radius from inclusive ep scattering at HERA, Phys.Lett. B 757 (2016) 468 [arXiv: 1604 . 01280].

[3] ZEUS Collaboration, H. Abramowicz et al., Limits on contact interactions and leptoquarks at HERA, Phys. Rev. D 99, no. 9 (2019) 092006 [arXiv: 1902.03048 ].

[4] W. Buchmuller, R. Ruckl and D. Wyler, Leptoquarks in Lepton - Quark Collisions, Phys. Lett. B 191 (1987) 442, Erratum: Phys. Lett. B 448 (1999) 320.

[5] A. Djouadi, T. Kohler, M. Spira and J. Tutas, (e b), (e t) Type Leptoquarks at e p Colliders, Z. Phys. C 46 (1990) 679.

[6] ZEUS Collaboration, H. Abramowicz et al., Search for first-generation leptoquarks at HERA, Phys. Rev. D 86 (2012) 012005 [arXiv: 1205. 5179].

[7] xFitter Developers' Team, V. Bertone et al., xFitter 2.0.0: An Open Source QCD Fit Framework, POS (DIS2017) 203 (2018).

[8] O. Turkot, K. Wichmann and A.F. Żarnecki, Simplified QCD fit method for BSM analysis of HERA data [arXiv:1606.06670].

[9] R. D. Cousins, Am. J. Phys. 63, 398 (1995). doi:10.1119/1.17901 